\title{
Judgment of duration relations: Simultaneous and sequential presentation
}

\author{
DWIGHT W. CURTIS and STANLEY J. RULE \\ University of Alberta, Edmonton, Alberta, Canada T6G 2 E9
}

\begin{abstract}
Four groups of subjects gave category ratings of total duration, average duration, or difference in duration of pairs of temporal intervals presented either simultaneously or successively. The results indicated that temporal information is combined in very different ways, depending on whether the members of the pairs must be monitored in parallel or sequentially. While judgments of successively presented intervals were reasonably consistent with the predictions of the appropriate linear models, judgments of simultaneously presented intervals were not. The latter judgments conformed more closely to the predictions of a vector sum model. These results appear to be in conflict with a model of duration perception recently proposed by Eisler (1975) based on the assumption that judgment of temporal intervals involves a simultaneous monitoring of two intervals even when the intervals to be compared are presented successively.
\end{abstract}

For the most part, studies of the psychophysical relation between subjective duration and objective time have yielded linear or slightly positively accelerated functions (Gregg, 1951; Ross \& Katchmar, 1951; Stevens \& Galanter, 1957). Most studies have employed the methods of ratio production and/or magnitude estimation, both of which require judgments of ratios, as a basis for scaling subjective duration. However, there are legitimate reasons for questioning whether these scales have the metric properties attributed to them (Attneave, 1962; Garner, 1954). Curtis and Rule and their associates (Curtis, Attneave, \& Harrington, 1968; Rule, Curtis, \& Markley, 1970) have presented evidence that the exponent of the empirical power function typically found to describe the relation between stimulus and response in the ratio scaling experiment may constitute a parameter of a phenotypical function that reflects the confounding of a nonlinear response function with the genotypical sensory relation.

In a recent study, Blankenship and Anderson (1976) circumvented the question of their scale's metric properties by employing a method that permitted a test of the metric assumptions. Their subjects were required to give graphic ratings of the total duration of two temporal intervals presented successively. Because the pairs of durations were chosen from a factorial design, an analysis of variance provided a test of fit of an additive model to the data. According to Blankenship and Anderson, if the interaction was not significant, the additive model

This research was supported by Grants A9582 and A0151 from the National Research Council of Canada. Requests for reprints should be sent to Dwight $W$. Curtis, Department of Psychology, Biological Sciences Building, University of Alberta, Edmonton, Alberta, Canada T6G 2E9. would be supported and the marginal means should constitute a scale of subjective duration with interval properties. In one of their experiments, the interaction was not significant, while in a second experiment it was slight, but significant. In both cases, the marginal means were related to measured duration by approximately linear functions.

In the initial experiment in the series to be reported, the strategy used was similar to that of Blankenship and Anderson, with an important exception. The stimuli were presented simultaneously rather than successively. It was decided to employ this variation in procedure because of issues associated with the successive presentation of two intervals, a feature common to most previous studies. Apparent duration is known to be subject to strong time-order errors (Woodrow, 1951), and these could be expected to determine at least in part judgment of the relation between stimuli. Furthermore, we were concerned that subjects who were instructed to estimate total duration, as in the Blankenship and Anderson study might estimate only a single interval, between the onset of the first interval and the offset of the second interval, rather than integrate the duration information from the two successively presented intervals. Blankenship and Anderson attempted to control for this possibility in one study by varying the length of the interstimulus interval. An analysis of variance yielded a nonsignificant $F$ for interstimulus interval, but it indicated a small interaction of this variable with the duration of the first stimulus (Anderson, Note 1).

Because the results of our initial experiment were not consistent with Blankenship and Anderson's data, three additional experiments were conducted. These were designed to determine whether the discrepancy was a result of the use of different composi- 
tion rules or was artifactual, a consequence, say, of a response bias in our experiment or the adoption by Blankenship and Anderson's subjects of a judgment strategy requiring the evaluation of only a single interval, as was suggested above. In the second experiment, subjects judged the average duration of two successively presented intervals. It was assumed that requiring judgments of averages rather than judgments of sums would reduce the probability that subjects would evaluate a single interval when stimuli were successively presented. The two remaining experiments were conducted to test the reliability of the results and to determine whether the findings could be generalized to judgments of a different relation. In these experiments, subjects judged either the average duration of simultaneously presented intervals or the difference in duration between successively presented intervals. The latter task would appear to guarantee that subjects integrate information concerning the two intervals in arriving at their response. We avoided judgments of difference in duration for simultaneous presentations because subjects could estimate the difference by evaluating a single interval, between the offset of the shorter interval and the offset of the longer interval.

\section{JUDGMENTS OF TOTAL DURATION: SIMULTANEOUS PRESENTATION}

\section{Method}

Subjects. Seventeen undergraduate students participated as subjects to fulfill a requirement of an introductory course in psychology.

Apparatus and Stimuli. The stimulus intervals were represented by two luminous red targets, controlled by electronic timers (Western Bioresearch, Model 500), which were triggered by a Hunter timer. Seven durations were selected, equally spaced over the range from 1.4 to $10 \mathrm{sec}$. For the judgments of total duration, these were combined according to a factorial design to generate a stimulus series of 49 pairs of durations.

Procedure. The subjects were tested in groups of three, except for the final group, in which there were only two subjects. On each trial, the pair of red targets were illuminated simultaneously, and subjects were instructed to rate the total duration of the two targets on a scale of 1 to 11 by recording their responses on a record sheet. The subjects were requested to give their global subjective impressions of the duration that would be produced if they were able to concatenate the two stimulus intervals. They were also cautioned to avoid counting or other strategies as an aid to judgment, and were asked to treat differences between the numerical category names as equal differences on their subjective scale. Ten practice trials were provided so as to familiarize subjects with the range of durations to be presented, and then each of the 49 stimulus combinations was presented twice in a pseudorandom order. A different order was presented to each of the groups of three subjects. Following the judgments of paired stimuli, each of the seven stimulus durations was presented individually four times in random order, and the subjects were requested to rate their magnitudes on a scale of 1 to 11 .

\section{Results}

The median judgments of total duration, pooled over replications and subjects, are plotted in Figure 1

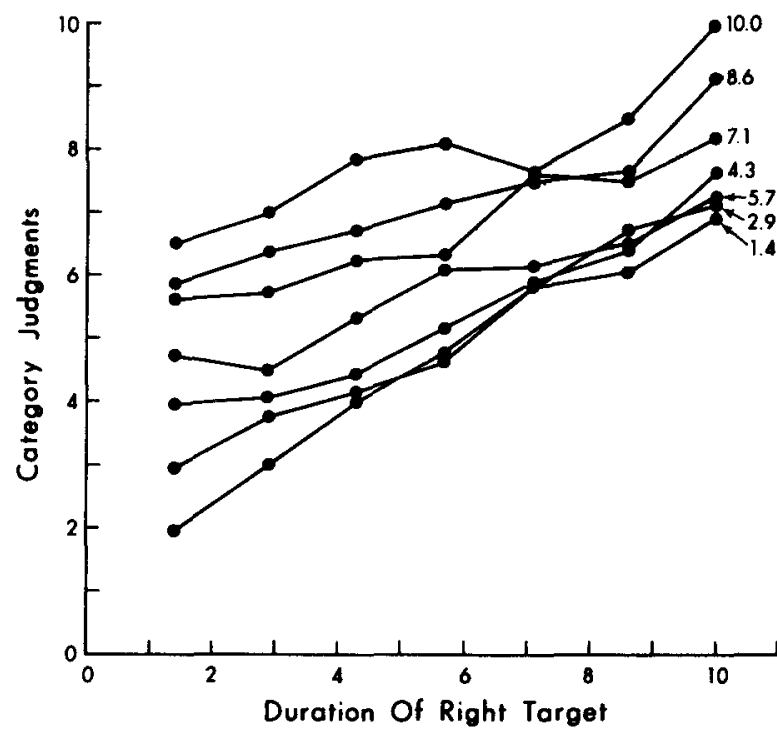

Figure 1. Category judgments of total duration, simultaneous presentation, as a function of the duration of the right-hand target. The parameter is the duration of the left-hand target.

as a function of the duration of the target on the right. Lines connect points associated with a constant duration for the left target. If the additive model held, consistent with instructions, the data should plot as a set of parallel functions. Obviously, this condition does not hold for these data; the functions tend to converge as the duration of the right target increases.

The results of an analysis of variance confirmed the interpretation that the curves tend to converge. In the experiment, subjects were tested in groups of three, each group receiving a different stimulus order. Consequently, the experiment employed a three-way, 6 by 7 by 7 factorial design in which groups (orders) constituted a between-subjects factor and left and right stimuli constituted within-groups factors. Because one subject was missing from one group (17 subjects participated in the experiment), an unweighted-means solution was used. The analysis resulted in a highly significant interaction between left and right stimuli $[F(36,180)=3.39$, $\mathrm{p}<.01$ ], consistent with the appearance of Figure 1 . Neither the main effect associated with groups nor the interactions implicating this factor were significant ( $F<1$, all tests). Apparently order effects did not exert an important effect upon the results.

The discrepancy between the present results and those of Blankenship and Anderson (1976) suggests that a different composition rule is applied in judging total duration when the stimulus durations are monitored simultaneously compared with when they are evaluated successively. However, there are at least two alternative interpretations that may also be considered. For instance, the interaction evident 
in the present data may merely reflect a response bias, such that subjects' ratings of total duration represent only an ordinal scale of their impressions of total duration. Second, the appearance of additivity in the results of Blankenship and Anderson may be an artifact of their procedure, and may not reflect the way in which duration information is combined. The latter interpretation is consistent with the earlier suggestion that in judging the total duration of sequentiaily presented temporal intervals, subjects might adopt a strategy of evaluating but a single interval, between the onset of the first interval and the offset of the second interval.

If the interaction was a consequence of a nonlinear bias, a scale of subjective duration may be recovered from the data by a nonmetric scaling solution. Such a solution requires only that judgments of total duration be monotone with subjects' subjective impressions of total duration. The scale so obtained should be related to those of Blankenship and Anderson by a linear relation, and consequently, should be approximately linear with measured duration. To test this hypothesis, the median ratings were subjected to UNICON (Roskam, Note 2), a nonmetric scaling algorithm for polynomial conjoint models. A solution for an additive model was obtained with a stress (Formula 2) of .12. (For this case, a solution from UNICON is within a linear transformation of one from Kruskal's, 1965, MONANOVA.) The scale values yielded by this solution are shown in Figure 2, plotted as a function of stimulus duration. This function is clearly concave upward, indicating that if an additive model is appropriate the psychophysical relation between subjective and objective duration is not linear. Clearly, the present results are not consistent with those of Blankenship and Anderson, and the discrepancy cannot be accounted for wholly on the basis of the assumption that nonlinear response biases were operating in one experiment but not the other.

The possibility that the apparent additivity exhibited by Blankenship and Anderson's data are artifacts of their method cannot be evaluated on the basis of the foregoing analyses. Consequently, a second experiment was conducted in which subjects were required to estimate the average magnitude of pairs of stimulus intervals that were presented successively.

\section{JUDGMENTS OF AVERAGE DURATION: SUCCESSIVE PRESENTATION}

\section{Method}

Subjects. Thirty-three undergraduate students participated in the experiment to fulfill a requirement of an introductory psychology course.

Stimulf. The stimuli consisted of eight temporal intervals: $.5,1.9,3.2,4.6,5.9,7.3,8.6$, and $10.0 \mathrm{sec}$. For the judgments

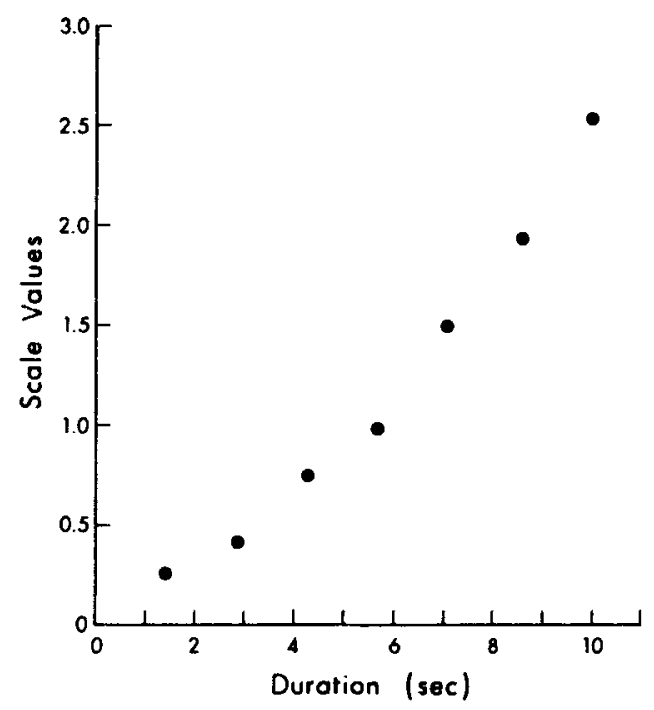

Figure 2. Scale values yielded by a nonmetric scaling solution plotted as a function of stimulus measures.

of average duration, these were combined according to an 8 by 8 factorial design to provide 64 combinations of durations.

Procedure. The subjects were tested in 11 groups of three subjects each. The procedure was the same as for the previous experiment, except that subjects were instructed to rate the average duration of the pairs on a scale of 1 to 11 . The members of the stimulus pairs were presented sequentially with the termination of the first interval triggering the onset of the second interval. Following the judgments of paired stimuli, subjects were presented with two series of each stimulus interval separately, and were asked to rate their magnitudes on a scale of 1 to 11 . Ten practice trials preceded each judgment task.

\section{Results}

The median ratings, pooled over replications and subjects, are plotted as a function of the measure of the second stimulus in Figure 3. The parameter is the measure of the first stimulus. The convergent interaction which characterized the data of the previous experiment is not evident in these data. The functions appear to be relatively parallel, although there is some appearance of constriction at intermediate values of the second stimulus. Nevertheless, there is a small, but significant, interaction between the factors associated with the first and second stimuli of the trial sequence: $F(49,490)=1.97$, $\mathrm{p}<.01$. It appears likely that it reflects the tendency noted in Figure 3 for the functions to converge in the midrange. A decomposition of the interaction into its bilinear and higher order components shows the bilinear component to be nonsignificant $[F(1,10)$ $=2.99, \mathrm{p}>.05]$ and the interaction to be largely concentrated in the residual $[F(48,480)=1.72$, $\mathrm{p}<.01]$. In other words, there is little of the tendency for the data to form a converging (as in our first experiment) or diverging fan of functions, as would be the case if the bilinear component con- 


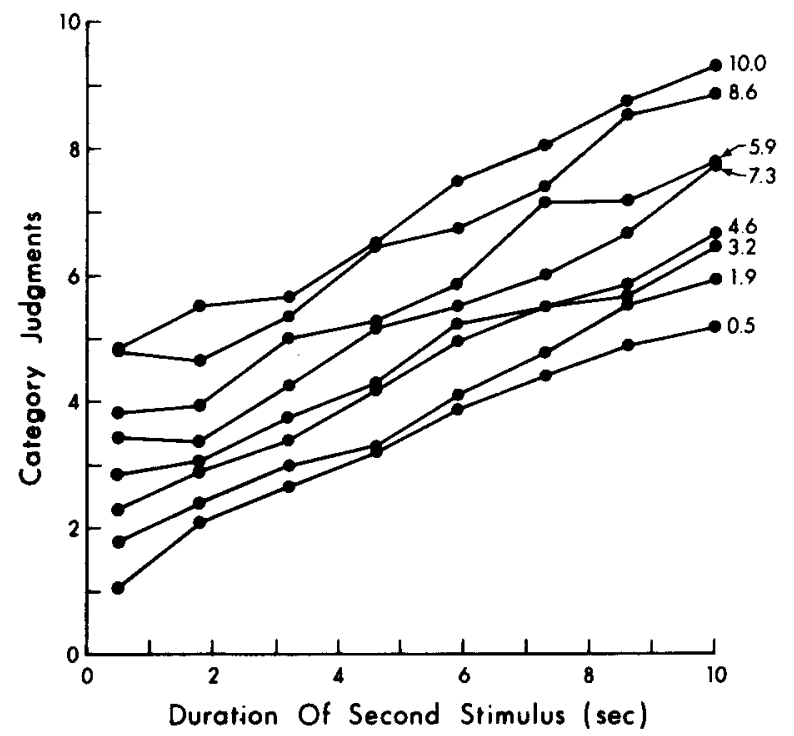

Figure 3. Category judgments of average duration, successive presentation, plotted as a function of measures of the second stimulus duration. The parameter is the first stimulus measure.

stituted a major portion of the interaction. The form of the interaction present in these data might be a consequence of time-order errors if the latter effects varied in magnitude and direction, as Woodrow (1951) suggested.

Effects of order are also indicated by significant interactions between groups and the first stimulus duration $[\mathrm{F}(70,140)=1.64, \mathrm{p}<.01]$ and groups and the second stimulus duration $[\mathrm{F}(70,140)=2.06$, $\mathrm{p}<.011$, and also by a significant three-way Groups by First Stimulus by Second Stimulus interaction $[F(490,1078)=2.44, p<.01]$. Why the order in which pairs of stimuli are presented should make a difference when the stimuli making up the pairs are presented successively but not when they were presented simultaneously is not clear.

Subjects' median judgments of average duration were also subjected to UNICON, the nonmetric scaling solution previously employed in the analysis of the data from judgments of total duration. A solution was obtained with a stress of .09. In Figure 4, the scale values yielded by this solution are plotted as a function of stimulus values. The relation is approximately linear, consistent with the finding of Blankenship and Anderson.

\section{DESCRIPTIVE MODELS FOR JUDGMENT OF THE CONJOINT EFFECT OF PAIRED TEMPORAL INTERVALS}

The results of the two foregoing experiments suggest that the integration of duration information from pairs of temporal intervals follows different composition rules, depending upon whether the information is processed sequentially or in parallel. In the case of judgments of the average duration of sequentially presented intervals, it would appear that the judgments are reasonably well described by an additive composition rule, that subjective duration is approximately a linear function of objective duration, and that subjective impressions of average duration are at least approximately linearly related to the average of the measured durations. With respect to judgments of the total duration of simultaneously monitored intervals, the case is somewhat more complex. Even when the judgment has been transformed to optimize its fit by an additive model, the nonmetric scaling analysis implies that the scale of subjective duration that would have generated it is not consistent with the scale from judgments of sequentially presented stimuli.

A general descriptive model for the relation between judgments and stimulus values that accounts for the main trends in the data can be specified. This model is given by the expression

$$
\mathrm{J}_{\mathrm{ij}}=\mathrm{a}\left[\mathrm{w} \phi_{\mathrm{i}}^{\mathrm{k}}+(1-\mathrm{w}) \phi_{\mathrm{j}}^{\mathrm{k}}\right]^{\mathrm{m}}+\mathrm{b},
$$

where $J_{i j}$ constitutes a judgment of the conjoint effect of the durations with measures $\phi_{i}$ and $\phi_{j}$, and $\mathrm{w}$ and $1-\mathrm{w}$ unequally weight the first and second stimuli of a series, reflecting the presence of timeorder errors. When the stimuli are presented simultaneously, it would be expected that $w=1-w$ $=.5$. Given that the composition rule is additive, and that subjective duration is a linear function of measured duration, the parameters $k$ and $m$ should each equal unity. If, as in the present judgments of total duration, a convergent interaction is present, $m$ constitutes the exponent of a power transformation which approximates the form of that interaction, and if the relation between scale values (say, from a nonmetric scaling solution) and objective duration is

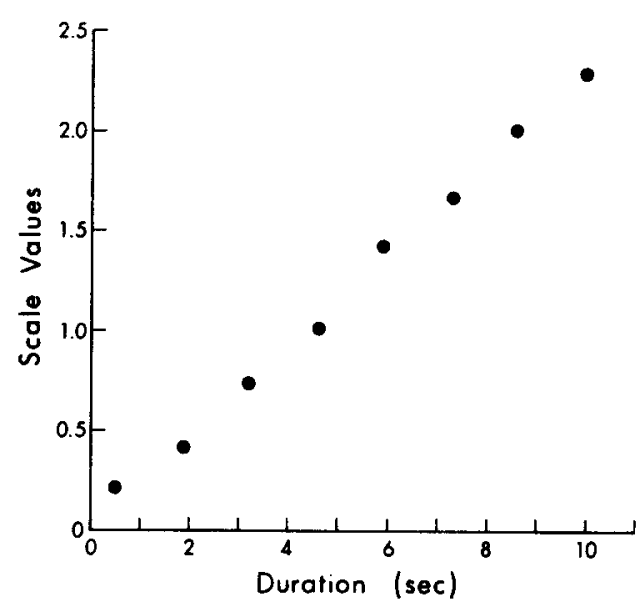

Figure 4. Scale values from a nonmetric solution as a function of stimulus duration. 
nonlinear, $\mathbf{k}$ represents the value of the exponent of a power function which best approximates that nonlinear relation.

Equation 1 was fitted to the relation between median judgments and stimulus values for the data of each of the two experiments by the Gauss-Newton method (Hartley, 1961). For the data from judgments of total magnitude of simultaneously presented temporal intervals, the best-fitting expression was provided by

$$
\mathrm{J}_{\mathrm{ij}}=.95\left(.51 \phi_{\mathrm{i}}^{1.94}+.49 \phi_{\mathrm{j}}^{1.94}\right)^{.49}+.94,
$$

while for judgments of average duration of successively presented stimuli a best fit was provided by

$$
\mathrm{J}_{\mathrm{ij}}=.53\left(.46 \phi_{\mathrm{i}}{ }^{1.09}+.54 \phi_{\mathrm{j}}{ }^{1.09}\right)^{1.08}+1.25 \text {. }
$$

The relations accounted for $97 \%$ and $98 \%$ of the variance, respectively. The weights for simultaneous presentations are approximately equal $(w=.51$, and $1-\mathrm{w}=.49$ ), while for successive presentations the difference was slightly greater, the second stimulus receiving somewhat more weight than the first $(w=.46$ and $1-w=.54)$. The difference is small, however, and requires further evidence for conformation. The major differences between the two sets of data appear in the estimates of $\mathrm{k}$ and $\mathrm{m}$. For judgments of total duration of simultaneously presented intervals, the estimate of $k$ is approximately two (1.94), and the estimate of $\mathrm{m}$ is close to one-half (.49). In contrast, the estimates of $\mathrm{k}$ and $\mathrm{m}$ for responses to successively presented stimuli are each close to unity $(\mathrm{k}=1.09$ and $\mathrm{m}=1.08)$, indicating an approximately linear psychophysical function for subjective duration and that the relation between subjective impression of average duration and the response is also close to linear.

In order to evaluate how robust the discrepancy between the results of simultaneous and successive monitoring of temporal intervals is, two additional conditions were evaluated. In the first task, subjects judged the average duration of intervals presented simultaneously, and in the second task, subjects judged the difference in duration of intervals presented successively.

\section{COMPOSITION RULES FOR SIMULTANEOUS AND SUCCESSIVE PRESENTATIONS}

\section{Method}

A triangular design was used in each experiment. The set of 8 stimuli used in the previous experiment were combined to form 28 combinations by pairing each stimulus interval with all others. In each task, the series was presented to each subject in random order three times. In addition, the individual stimuli were presented three times and subjects were required to rate their magnitudes. The procedure was the same as was used in the previous experiments. Thirty subjects judged the average magnitudes of simultaneously presented durations, and 29 subjects judged differences between successively presented durations.

\section{Results}

The data of each experiment were pooled over replications and subjects by calculating medians, and expressions were fitted to the medians by the Gauss-Newton method. For the judgments of average duration, the model fitted to the data is given by the expression

$$
\mathrm{J}_{\mathrm{ij}}=\mathrm{a}\left(\phi_{\mathrm{i}}^{\mathrm{k}}+\phi_{\mathrm{j}}^{\mathrm{k}}\right)^{\mathrm{m}}+\mathrm{b} .
$$

This expression differs from Equation 1 only in that the two stimuli constituting each pair are equally weighted. There appeared to be little justification for weighting stimuli unequally when they were presented simultaneously, and the results from judgments of total duration of simultaneously presented intervals suggested that under these conditions subjects do in fact weight the stimuli equally. Consequently, it seemed advisable to employ Equation 2, which required that one fewer parameter be estimated.

A least-squares fit was provided by the expression

$$
\mathrm{J}_{\mathrm{ij}}=.55\left(\phi_{\mathrm{i}}{ }^{1.85}+\phi_{\mathrm{j}}{ }^{1.85}\right)^{.52}+.97
$$

which accounted for $99.2 \%$ of the variance. The estimated value of 1.85 for $k$ is slightly smaller than that obtained from judgments of total duration, but considerably larger than was obtained from judgments of the average duration of successively presented intervals. The estimated value of $\mathrm{m}$ is roughly one-half, consistent with the exponent obtained from judgments of total duration.

The model fitted to judgments of difference is given by Equation 1 except that subtraction was substituted for addition. Because the stimuli were presented sequentially, weights are retained to account for the presence of any order effect. A leastsquares fit to the data was provided by the expression

$$
\mathrm{J}_{\mathrm{ij}}=1.27\left[.47 \phi_{\mathrm{i}}^{1.0}-.53 \phi_{\mathrm{j}}^{1.0}\right]^{1.11}+.87 .
$$

The relation accounts for $97.9 \%$ of the variance. This result is essentially the same as that for judgments of average duration of sequentially presented intervals. It implies a linear psychophysical relation between subjective and objective time, and only very slightly curvilinearity between subjective impressions of difference and objective differences in duration. The difference between the weights associated with the two stimuli is small, but it is consistent with the previous result in implying that the second stimulus of a series is more heavily weighted than the first stimulus. 


\section{RELATION BETWEEN RATINGS OF STIMULUS MAGNITUDE AND OF STIMULUS RELATIONS}

In each of the foregoing experiments, judgments of the duration of single intervals were collected after the judgments of the combined effect of two temporal intervals. According to the model that was considered, the expected relation between judgments of single-stimulus durations and stimulus measures would constitute a case where $\phi_{j}$ is zero, and consequently,

$$
\mathbf{J}=\mathbf{a} \phi^{\mathrm{km}}+\mathrm{b} .
$$

Previous research has shown that the relation between category ratings and stimulus measures is well described by a power function (Curtis, 1970; Marks, 1968) of the form

$$
\mathrm{J}=\mathbf{a} \phi^{\mathrm{n}}+\mathbf{b} .
$$

Equations 3 and 4 imply that the exponent $n$ can be partitioned into two components, such that $n=k m$. If such is the case, the value of $n$ can be estimated in two ways: by fitting Equation 4 directly to the relation between category ratings and stimulus measures, and by calculating the product of $k$ and $m$ estimated by fitting Equation 1 or Equation 2 to judgments of paired durations.

The Gauss-Newton method was employed to fit Equation 4 to the relation between category ratings of each duration and its corresponding stimulus measure for the data collected in each of the four experiments. The least-squares estimates of $n$ are listed in Table 1, together with estimates from the product of $k$ and $m$. Note that in each case the estimate from the product of $\mathrm{k}$ and $\mathrm{m}$ is quite close to that obtained from an independent task.

It is surprising that $n$ is predicted about equally well from the product of $k$ and $m$ for estimates obtained from both simultaneously presented pairs and successively presented pairs. This is paradoxical because the values of $k$ and $m$ are very different in the two cases. One should note, however, that the predicted $\mathrm{n}$ is approximately the same if $\mathrm{k}$ and $\mathrm{m}$ are equal to 1 (resulting in $\mathrm{n}=1$ ), or if $\mathrm{k}$ is equal to 2 and $m$ equals one-half (so that $n=1$ ), which seems to approximate the present case.

\section{DISCUSSION}

The results clearly demonstrate that which composition rule subjects employ in integrating duration information from two temporal intervals depends on whether the stimulus intervals are monitored simultaneously or successively. The data are con-
Table 1

Relation Between Parameters of Integration Model and Exponents of Power Function Fitted to Category

\begin{tabular}{|c|c|c|c|c|c|}
\hline & \multicolumn{5}{|c|}{ Parameter } \\
\hline & $\mathbf{w}$ & $\mathrm{k}$ & $\mathrm{m}$ & $\mathrm{k} \times \mathrm{m}$ & $\mathrm{n}$ \\
\hline & \multicolumn{5}{|c|}{ Simultaneous } \\
\hline \multirow[t]{2}{*}{$\begin{array}{l}\text { Total Duration } \\
\text { Average Duration }\end{array}$} & & $\begin{array}{l}1.94 \\
1.85\end{array}$ & $\begin{array}{l}.49 \\
.52\end{array}$ & $\begin{array}{l}.95 \\
.96\end{array}$ & $\begin{array}{l}.94 \\
.97\end{array}$ \\
\hline & \multicolumn{5}{|c|}{ Successive } \\
\hline $\begin{array}{l}\text { Average Duration } \\
\text { Duration Differences }\end{array}$ & $\begin{array}{l}.46 \\
.47\end{array}$ & $\begin{array}{l}1.09 \\
1.00\end{array}$ & $\begin{array}{l}1.08 \\
1.11\end{array}$ & $\begin{array}{l}1.16 \\
1.11\end{array}$ & $\begin{array}{l}1.19 \\
1.04\end{array}$ \\
\hline Mean & & & & 1.04 & 1.04 \\
\hline
\end{tabular}
Judgments of Duration

sistent with a linear composition rule and a linear psychophysical function when the information to be integrated was presented sequentially. But, for simultaneous presentations, a more complex way of processing duration information is reflected in the interaction that was found to characterize the data. An interesting aspect of the relation between results from simultaneous and successive presentations is suggested by the analysis in terms of Equations 1 and 2 . The value of the exponent $k$ estimated from judgments of simultaneously presented pairs is approximately twice as large as its estimate from judgments of successively presented pairs, while the estimate of the exponent $m$ is only about one-half as great. This would suggest that if subjective duration is related to measured duration by a linear function, as was indicated by the data from successively presented stimuli, judgments of simultaneously monitored intervals are based on a composition rule of the form

$$
\psi_{\mathrm{ij}}=\left(\psi_{\mathrm{i}}^{2}+\psi_{\mathrm{j}}^{2}\right)^{1 / 2},
$$

where $\psi_{\mathrm{ij}}$ represents the subjective measure of the conjoint effect of stimuli $\mathrm{i}$ and $\mathrm{j}$, and $\psi_{\mathrm{i}}$ and $\psi_{\mathrm{j}}$ represent the subjective magnitudes of the durations that were evaluated. That is, the subjective impression of the total magnitude (or average) is represented by a vector sum in which the magnitude of each vector represents the subjective magnitude of the separate intervals.

Equation 5 represents a special case of vector summation in which the angle separating the two vectors is $90^{\circ}$, i.e., when the vectors are orthogonal. The model implies that summation is less than complete and that stimuli with greater magnitudes are weighted more heavily than stimuli of lesser magnitudes. Vector sum models have been proposed by Berglund, Berglund, and Lindvall (1976) to account for the conjoint effects of the intensities of odorants making up an odor mixture, and by Curtis and Rule (1978) to describe the interaction between monocular 
visual channels in the binocular processing of brightness information. However, these cases differ from the present one in that the interaction for odor mixture and binocular brightness is probably a function of events within the respective sensory nervous systems, whereas in the present case the interaction is more likely cognitive in origin. Also, the values of the parameters of the vector models considered by Berglund et al. and by Curtis and Rule imply a composition rule in which partial summation is observed when the subjective intensities of the stimuli that are combined are nearly equal, but the resultant is intermediate between the subjective intensities when they are very different. The present model does not exhibit the latter property.

The present result appears to have a bearing upon some theoretical issues raised by Eisler (1975). He argued for a model for which the essential assumption is that when standard and comparison intervals are serially presented, comparison is based on a simultaneous monitoring of two intervals, the total duration from the onset of the standard to the offset of the comparison interval and the duration of the comparison stimulus. The evaluation of the magnitude of the standard duration is assumed to be based on a "calculation" of the difference between the total duration and the comparison interval.

The present result appears to contradict Eisler's interpretation. If it is correct that the evaluation of the subjective durations of a pair of intervals is based on a simultaneous monitoring process regardless of whether the durations making up the pairs are presented simultaneously or successively, there is little reason to expect that the composition rule defining the way in which duration information is combined should be different. We have seen that, in fact, they are.

Finally, both the present results and those of Blankenship and Anderson (1976) point up an apparent inconsistency relevant to the interpretation of category rating scales and scales based on ratio judgments obtained from direct estimates of relative magnitude. Most attempts to scale subjective time have employed ratio scaling procedures, such as fractionation or magnitude estimation. For the most part, these investigations have resulted in scales that are related to measured duration by approximately linear functions or by power functions with exponents very near unity. Typically, category rating scales have been found to be related to scales based on ratio judgments by a nonlinear, negatively accelerated, function. Consequently, it is surprising that the results obtained by Blankenship and Anderson and those obtained in the present investigation are also consistent with a psychophysical relation that is approximately linear, or is described by a power function with an exponent very close to unity.

\section{REFERENCE NOTES}

1. Anderson, N. H. Personal communication, July 11, 1977.

2. Roskam. E. E. Unidimensional conjoint measurement (UNICON) for multi-faceted designs. Report 74 MA 09, Psychologisch Laboratorium, Universiteit, Nijmegen. The Netherlands.

\section{REFERENCES}

Attneave, F. Perception and related areas. In S. Koch (Ed.), Psychology: A study of a science (Vol. 4). New York: McGraw-Hill, 1962.

Berglund, B., Berglund, U., \& Lindvall, T. Psychological processing of odor mixtures. Psychological Review, 1976, 83, $432-441$.

Blankenship. D. A.. \& Anderson, N. H. Subjective duration: A functional measurement analysis. Perception \& Psychophysics. 1976. 20. 168-172.

Curtis, D. W. Magnitude estimations and category judgments of brightness and brightness intervals: A two-stage interpretation. Journal of Experimental Psychology, 1970, 83. 201-208.

Curtis, D. W., Attneave, F., \& Harrington. T. A test of a two-stage model of magnitude estimation. Perception \& Psychophysics. 1968, 3, 24-31.

Curtis. D. W., \& Rule. S. J. The binocular processing of brightness information: A vector-sum model. Journal of Experimental Psychology: Human Perception and Performance. 1978,4 , in press.

Eisler, H. Subjective duration and psychophysics. Psychological Review, 1975. 82, 429-450.

GARNER. W. R. A technique and a scale for loudness measurement. Journal of the Acoustical Society of America, 1954, 26. $73-88$.

GREGG, L. W. Fractionation of temporal intervals. Journal of Experimental Psychology, 1951, 42. 307-312.

Hartley, H. O. The modified Gauss-Newton method for the fitting of nonlinear regression functions by least squares. Technometrics, 1961, 3, 269-280.

KRUSXAL, J. B. Analysis of factorial experiments by estimating monotone transformations of the data. Journal of the Royal Statistical Society (B), 1965, 27, 251-263.

Marks, L. E. Stimulus-range, number of categories, and form of the category-scale. American Joumal of Psychology, 1968. 81, 467.479.

Ross, S., \& Katchmar, L. The construction of a magnitude scale for short time intervals. American Journal of Psychology, 1951, 64, 397.401.

Rule, S. J., Curtis, D. W., \& Markiey, R. P. Input and output transformations from magnitude estimation. Journal of Experimental Psychology, 1970, 86, 343-349.

Stevens. S. S.. \& Galanter. E. Ratio scales and category scales for a dozen perceptual continua. Journal of Experimental Psychology, 1957, 54, 377-411.

Woodrow, H. Time perception. In S. S. Stevens (Ed.), Handbook of experimental psychology. New York: Wiley, 1951.

(Received for publication June 13, 1977; revision accepted September 21, 1977.) 